\title{
A Vessel Flow Detection System based on Laser Sensors
}

\author{
http://dx.doi.org/10.3991/ijoe.v10i2.3427 \\ W.Q. Li, Y.T. Yang and S.H. Shen \\ Nanjing University of Science and Technology, Nanjing, China
}

\begin{abstract}
A system using two laser sensors for vessel flow detection was introduced. Mathematical morphology on ship contour detection was studied and vessel's contour was reconstructed based on geometric space projection, vessel's width threshold filtering and connected graph merging. Also, the necessity of vessel's contour reconstruction is verified by experiments. A data fusion method based on two laser sensors was researched for vessel's length detection, which using spatial adjustment and data correlation to calculate the speed, and to estimate the length of a vessel. Finally, a vessel flow detection system was built and worked fine.
\end{abstract}

Index Terms - vessel flow, laser sensor, feature extraction, mathematical morphology, data fusion

\section{INTRODUCTION}

The Beijing-Hangzhou Grand Canal makes a great influence on the city along the Canal and even the economy of the whole country because of its huge traffic. The pressure of transportation is becoming greater because of the ever-growing traffic in the Canal. So now the problem of shipping is becoming serious. To improve the safety of shipping, the department of navigation and the maritime agency must strengthen supervision. And at first, they must solve the problem of real-time detection of vessel flow. It has too many constrains for detection vessel flow using normal technologies, and a practical way need to be found.

At present, the method to get ship information mainly includes radar imaging system, video monitor system, infrared imaging system, AIS monitoring system and so on. Radar can be disturbed easily by wave and building, video monitoring can be used only in the daytime, infrared imaging system need artificial auxiliary and AIS monitor- ing need to be installed in the ship. A method based on laser sensors to detect the ship is proposed because of laser sensor is great anti-disturbing, high-accuracy and has high sampling frequency. The laser sensors can accomplish the real-time detection. Also the weather has a small influence on laser, so it can work all day.

\section{The Principle of Vessel Detection}

This system extracts the ship features by means of the combination of Laser sensor LD-LRS3100 and LMS221. The features extracted include length, width, speed, empty weight, height above water and length, width, height above water are the most important. (Figure 1.)

Laser sensor LD-LRS3100 scans the ship in the horizontal plane which is perpendicular to the water. Laser sensor LMS221 scans the front and bottom of the ship at an angle. Laser sensor LD-LRS3100 mainly detects the section of the ship to get the width and height above water of the ship and Laser sensor LMS-221 mainly detects the speed of the ship. So we can use both of them to get more perfect results.

\section{FEATURES EXTRACTION BASED ON MORPhOLOGY}

\section{A. The Ship Contour Detection}

The characteristic value of the vessel needs to be extracted, so it makes a great difference to the experimental results whether the painted ship contour can reflect the ship itself better. Although laser has a high scanning frequency, in fact we can't get a continuous contour because of all kinds of accident such as uncertain reflection, shading, etc. On the other hand, it always has deviation as we mistake some points which are made by the wave as ship. And these problems must be solved in the stage of contour detection and mathematical morphology is a good choice.

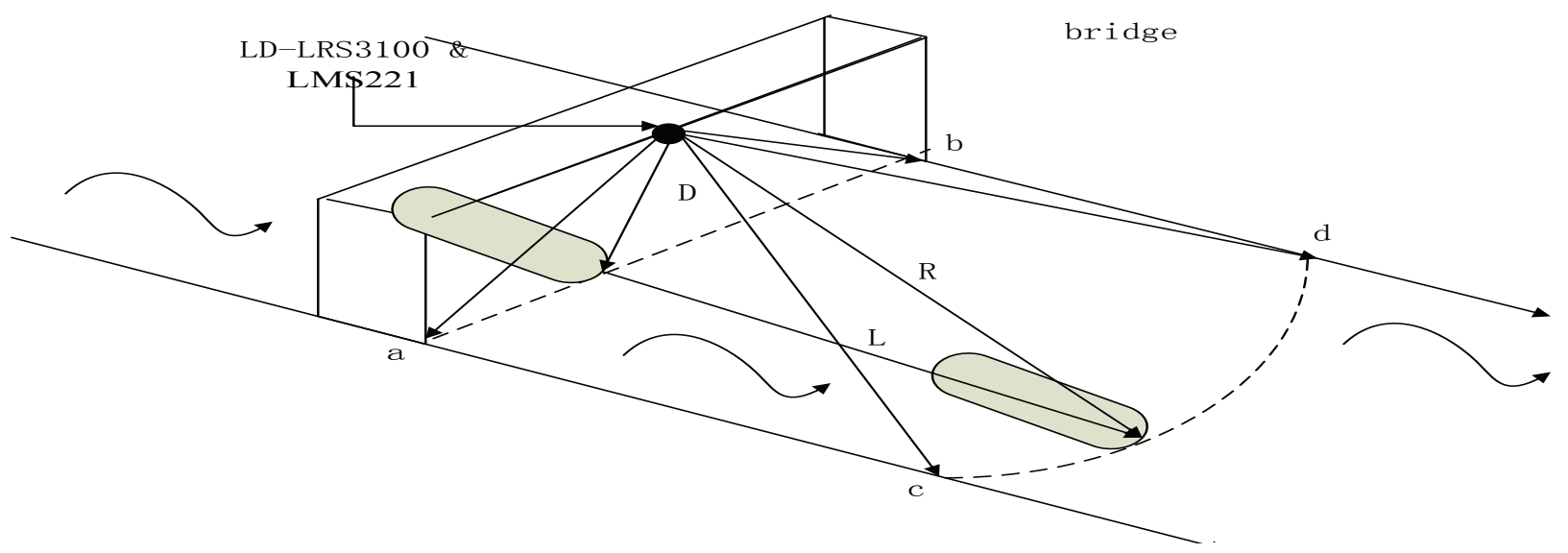

Figure 1. Principal of Vessel Detection 


$$
\begin{gathered}
\text { Define } X, Y \in Z^{2}, X \oplus Y \text { as } Y \text { expands } X \text { : } \\
X \oplus Y=\{x:(-Y+x) \cap X \neq \varnothing\}
\end{gathered}
$$

Expand $\mathrm{X}$ by operating $\mathrm{Y}$ to change $\mathrm{X}$ to get our final results.

Figure.2.(a) shows a picture of ship contour without water line. While we compute dilation, it matters that we could make an appropriate structural element. Different structural element has different impact on the same image. When we choose a structural element, the shape and size of the structural element matter. After overall consideration, we make a square whose side-length is 3 as our structural element. Figure.2(b). shows results of ship after expanding.

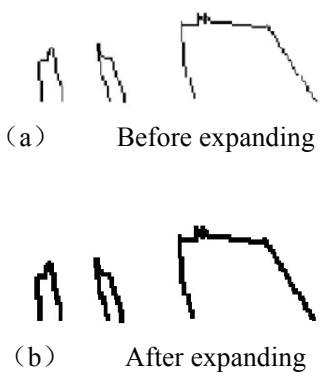

Figure 2. Ship Contour without Water Line

To compute how many boats there are in the current frame image, connected areas were identified using the Eight Chain Code Algorithm.

To make sure no mistake the wave and any kind of noise as ship, must filter out unnecessary data as following steps:

Scan binary-image to get the number of pixel in every connected region in terms of the definition of connected region.

Add the number of pixel in every connected region to the array accordingly.

Compare the number of pixel in every connected region to the given threshold in sequence. If it is bigger than the given threshold, then keep this region and otherwise delete it(here we set the threshold 0 .

After identifying the image in Figure.2(b) using the Eight Chain Code Algorithm, the 3 contours in the picture are coded No.1, No.2, No.3 from left to right.

\section{B. Reconfiguration of Ship Contour}

The necessity of reconfiguration of ship contour includes

1. Some ships sail just at one side and always have a higher height above water. At this time the laser can just scan one side of the ship and the other side always can't be seen, as Figure 3..

2. When a ship without ceil is empty, its bottom will be close to the water line and unfortunately we always delete it by mistake it as the water line. As a result, the ship is divided into two (Figure 4).

If we don't reconstruct ship contour, the first situation will get a bigger width of ship than the real. The second situation will get a lower number and smaller width of ship and this doesn't fit the fact.

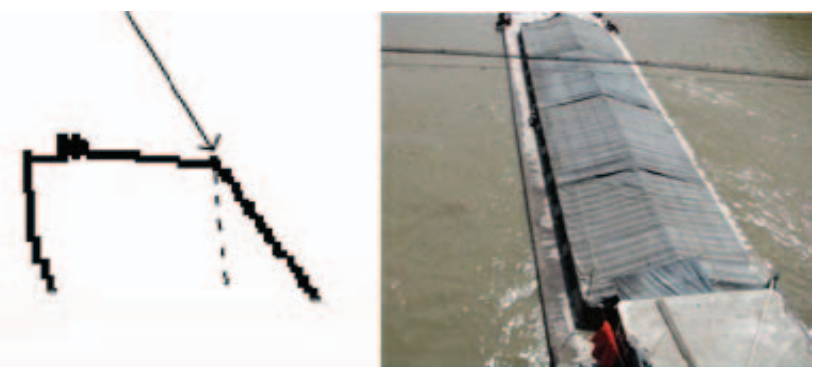

Figure 3. A ship just have one side

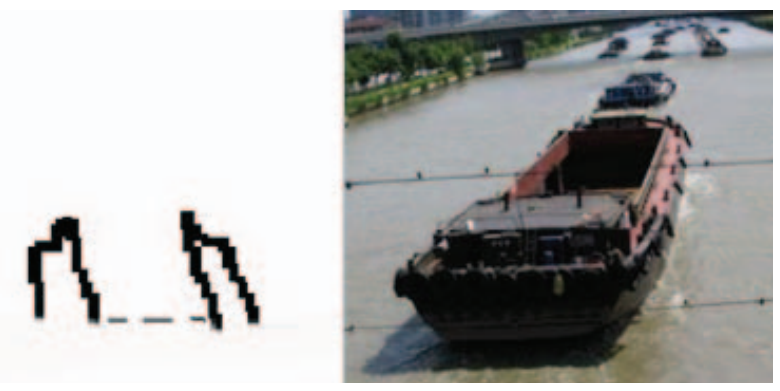

Figure 4. A ship is divided into two

In the first situation, we get a model of reconfiguration of ship. And we assume row $w_{\text {water }}$ as the row where water line locates, $\operatorname{row}_{L}$ and $\mathrm{col}_{L}$ as the row and the column respectively where leftmost point locates, $\operatorname{row}_{R}$ and $\mathrm{COl}_{R}$ as the row and the column respectively where rightmost point locates, row $w_{\text {top }}$ as the row where the top point locates. If all the parameters above subject to:

$\left|\operatorname{row}_{L}-\operatorname{row}_{\text {water }}\right|<\varepsilon$ Or $\left|\operatorname{row}_{L}-\operatorname{row}_{\text {water }}\right|<\varepsilon$

Then we only can get one side of the ship(left or right). Figure. 5 shows that the square acde is one contour and every side of square subject to:

$$
\left\{\begin{array}{l}
L_{a b}=L_{a c}-L_{b c} \\
L_{a c}=\left(c o l_{R}-\operatorname{col}_{L}\right) \lambda_{1} \\
L_{b c}=H \tan \theta \\
H=L_{d b} \approx\left(\text { row }_{\text {top }}-\text { row }_{\text {water }}\right) \lambda_{2}
\end{array}\right.
$$

We can get the actual width based on the following formular:

$$
W=\left(\mathrm{col}_{R}-\mathrm{col}_{L}\right) \lambda_{1}-\left(\text { row }_{\text {top }}-\text { row }_{\text {water }}\right) \lambda_{2} \tan \theta
$$

Where $\theta$ is dip angle when laser arrive at $\mathrm{d}, \lambda_{1}$ and $\lambda_{2}$ is actual distance between two neighbored pixels in every row and column respectively.

In the second situation where the ship is divided into two, we should check out whether the width of ship is exceptional as the following formula: 


$$
\left|\operatorname{col}_{R}-\operatorname{col}_{L}\right| \lambda_{1}<\frac{W_{\min }}{2}
$$

Where $\lambda_{1}$ is the $\lambda_{1}$ in Formula.(3) and Wmin is the given minimum-width in the system. Figure 6 shows a ship which is divided into ship. 1 and ship.2. The width of ship is fixed as following steps:

At first we will scan the image and compute the width of one part of connected region.

Then we will check out whether this width is compatible to the set value. If not, then we will make a mark on this part.

After then, we will keep on scanning the rest of the image and do the same thing like step(2).

At last we will get the two parts together and get the actual width of the ship

We can get the actual width of ship as the following formula:

$$
W=\frac{\left(\operatorname{col}_{d}+\operatorname{col}_{c}\right)-\left(\operatorname{col}_{b}+\operatorname{col}_{a}\right)}{2} \times \lambda_{1}
$$

\section{Experiment Results}

Experiments were performed on April 5th, 2012, and table 1 shows partial results. First define the mean error of width and height,

$$
\left\{\begin{array}{l}
\bar{\varphi}_{\mathrm{W}}=\frac{1}{n} \sum_{i=1}^{n} \frac{\left|W_{\text {mani }}-W_{\text {laseri }}\right|}{W_{\text {mani }}} \\
\bar{\varphi}_{H}=\frac{1}{n} \sum_{i=1}^{n} \frac{\left|H_{\text {mani }}-H_{\text {laseri }}\right|}{H_{\text {mani }}}
\end{array}\right.
$$

Here $\bar{\varphi}_{W}$ and $\bar{\varphi}_{H}$ is the mean error of width and height above water respectively. $(x, y)$ and $W_{\text {mani }}$ is the width of the ship measured by man and laser respectively. $H_{\text {mani }}$ and $H_{\text {laseri is the height above water of the ship }}$ measured by man and laser respectively.

According to formula (6), the error of width and height above water is $24.68 \%$ and $2.20 \%$ respectively where we don't reconstruct ship contour. But after reconfiguration, the error is $1.58 \%$ and $2.20 \%$ respectively. For width of ship, the error that we don't reconstruct ship contour is too big to accept it but the error that we reconstruct ship contour can be satisfactory. And for the height above water, there is no difference between these two kinds of situations. So Experiments show the necessity to reconstruct the ship contour.

\section{LENGTH EXTRACTION BASED ON DATA FUSION}

Laser sensor LD-LRS3100 was used to extract the weight and height above water of the ship. And in this section, a method is discussed to extract the length of ship and to finish this job, we need to deal with the data based on data fusion and the data is come from two laser. Figure 7. shows process to extract length of ship.

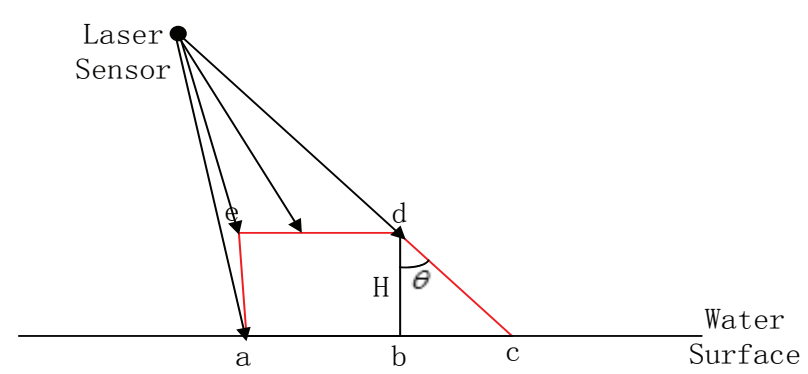

Figure 5. Vessel contour reconstruction based on geometry space projection

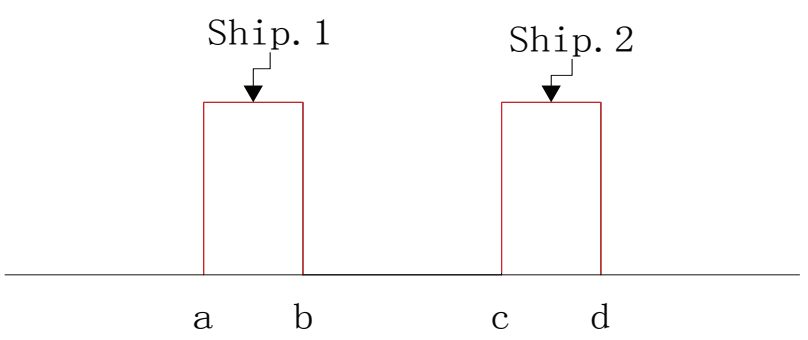

Figure 6. The ship is divided into two

TABLE I.

EXPERIMENT RESULTS

\begin{tabular}{|c|c|c|c|c|c|}
\hline Laser & Laser & Laser & Laser & Man & Man \\
\hline $\boldsymbol{O}-\boldsymbol{W}$ & $\boldsymbol{O}-\boldsymbol{H}$ & $\boldsymbol{R}-\boldsymbol{W}$ & $\boldsymbol{R}-\boldsymbol{H}$ & $\boldsymbol{W}$ & $\boldsymbol{H}$ \\
\hline 8.8 & 0.8 & 6.8 & 0.8 & 6.7 & 0.7 \\
\hline 9.7 & 1.6 & 8.7 & 1.6 & 8.6 & 1.5 \\
\hline 2.1 & 1.1 & 7.3 & 1.1 & 7.5 & 1.0 \\
\hline 1.8 & 1.7 & & & & \\
\hline 6.7 & 1.7 & 6.7 & 1.7 & 6.8 & 1.6 \\
\hline 7.8 & 1.0 & 7.8 & 1.0 & 7.7 & 1.0 \\
\hline 9.4 & 1.4 & 7.0 & 1.4 & 7.0 & 1.5 \\
\hline 2.1 & 2.0 & 7.8 & 2.0 & 8.0 & 2.0 \\
\hline 2.0 & 1.0 & & & & \\
\hline 9.2 & 1.0 & 9.2 & 1.0 & 9.0 & 1.0 \\
\hline 9.8 & 2.0 & 9.8 & 2.0 & 9.8 & 2.0 \\
\hline 8.4 & 0.6 & 8.4 & 0.6 & 8.5 & 0.6 \\
\hline
\end{tabular}

$\mathrm{O}-*$ means * is original

$\mathrm{R}-*$ means $*$ is data after reconfiguration

\section{A. Space Matching}

In the system of ship features extraction, the laser sensor used for tracking target measures the ship in CScoordination system. In many cases, CS is spherical coordinates in 3D or polar coordinates system in 2D. But Laser LMS221 works in spherical coordinates in 3D and Laser sensor LD-LRS3100 works in polar coordinates system in 2D. Obviously, to track the same target, we must transform CS-coordinate system into Cartesian coordinate system.

Now for Laser sensor LD-LRS3100 and LMS221, we will do projective transformation in $2 \mathrm{D}$ and $3 \mathrm{D}$ respectively. we establish a polar coordinate system in space of Laser sensor LD-LRS3100. The launching point of laser will be pole in coordinate and the straight line where the 0th scanning line locates will be pole axis. In this coordinate system, the coordinate value of any observation point is 


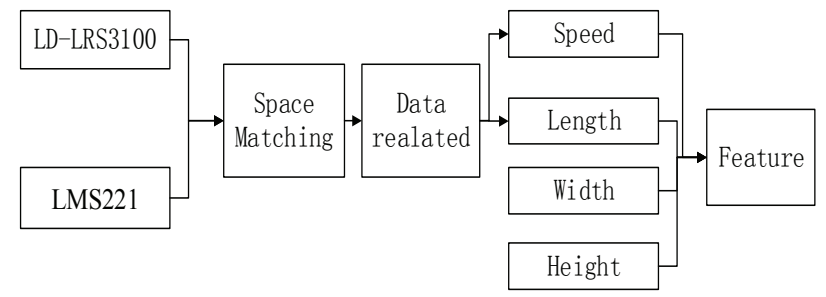

Figure 7. Detection Process

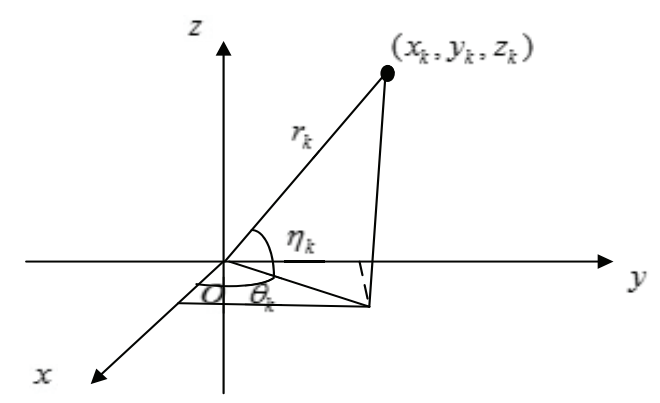

Figure 8 .

Figure 9. Projective Transformation in 3D

$(D, \theta)$ where $D$ is the distance value of point and $\theta$ is the angle value.

At the same time, we will establish the Cartesian coordinate system of the laser. The launching point of laser will be the origin. The coordinate of any point $\mathrm{p}$ is $(\mathrm{x}, \mathrm{y})$. And we will transform the polar coordinate system into the Cartesian coordinate system after we get the data.Then we will establish the spherical coordinates of Laser LMS221 in space and transform the coordinate into rectangular coordinate system(Figure 8.) and we will do this transformation as the following formula:

$$
\left\{\begin{array}{l}
x_{k}=r_{k} \cos \eta_{k} \cos \theta_{k} \\
y_{k}=r_{k} \cos \eta_{k} \sin \theta_{k} \\
z_{k}=r_{k} \sin \theta_{k}
\end{array}\right.
$$

\section{B. Data Correlation based on Multisensor}

We measure the speed of the ship by the combination of Laser sensor LD-LRS3100 and LMS221. According to Figure.1, when the ship arrives at line ab, Laser sensor LD-LRS3100 scans the ship and records the time $\mathrm{t} 1$ and the location of ship $x_{L}$ and when the stem of the ship cross the line, the Laser will record the time $\mathrm{t} 2$. And when the ship arrives at curve CD, the Laser sensor LMS221 will scan the ship and record the time $\mathrm{t} 3$ and the location of the ship $x_{R}$.

From formula (13) we can get $D^{2}$ is a normalized random variable. When X2-X1and Y2-Y1 in formula (13) submit to normal distribution, $D^{2}=x_{\text {submit to the }} \chi^{2}$ distribution whose free degree is 2 .
The two lasers are tracking the same ship when the deviation between $x_{L}$ and $x_{R}$ and the deviation between $\mathrm{t} 3$ and $\mathrm{t} 1$ is less than the given threshold. And we can get the velocity(8) and the length (9):

$L=\sqrt{R^{2}-D^{2}}, \quad v=\frac{L}{t_{3}-t_{1}}$

$l=v \times\left(t_{2}-t_{1}\right)$

Where $\mathrm{L}$ is the distance that the ship went at time between $\mathrm{t} 1$ and $\mathrm{t} 3$. When detecting the ship, Laser sensor LD-LRS3100 get the contour in 2D coordinate and Laser sensor LMS221 measure the speed in 3D coordinate. But in ship-related, it just can use 2D coordinate.

Assume the two measured normalized statistics distance which have been regulated in both time and space as:

$$
D^{2}=A^{T} S^{-1} A
$$

Where A-Matrix of observation error, S-Matrix of error covariance

If the coordinate $(X 1, Y 1)$ observed by Laser sensor LDLRS3100 is correspond to the predictor and $(X 2, Y 2)$ is the coordinate observed by Laser sensor LMS221. So we can get Matrix A:

$$
A=\left[\begin{array}{c}
X_{2}-X_{1} \\
Y_{2}-Y_{1}
\end{array}\right]
$$

Assume the random error of X2-X1 and Y2-Y1 are independent and the mean value is 0 and variance is $\sigma_{X}^{2}$ and $\sigma_{Y}^{2}$ respectively. So we can get the $\mathrm{S}$ and its inverse matrix:

$$
S=\left[\begin{array}{cc}
\sigma_{X}^{2} & 0 \\
0 & \sigma_{Y}^{2}
\end{array}\right] \quad S=\left[\begin{array}{cc}
\sigma_{X}^{2} & 0 \\
0 & \sigma_{Y}^{2}
\end{array}\right]
$$

put (11), (12), into formula (10) and we can get:

$D^{2}=\left[\begin{array}{c}X_{2}-X_{1} \\ Y_{2}-Y_{1}\end{array}\right]^{T}\left[\begin{array}{cc}\frac{1}{\sigma_{X}^{2}} & 0 \\ 0 & \frac{1}{\sigma_{Y}^{2}}\end{array}\right]\left[\begin{array}{c}X_{2}-X_{1} \\ Y_{2}-Y_{1}\end{array}\right]$

$f(x)=\frac{1}{2 \Gamma(1)} \exp \left(-\frac{x}{2}\right)$ 
According to the fundamental theorem of $\chi^{2}$, we can know that if random variable $D^{2}$ is less than critical value $\chi_{\alpha}^{2}$, then we can say the experiment is successful, otherwise we think the experiment fails. If successful, that means another data can enter into the correlation and the probability is:

$$
P=\int_{0}^{x_{\alpha}^{2}} f(x) d x
$$

And probability that the data can't enter into the correlation is:

$$
P_{G}=\int_{\chi_{\alpha}^{2}}^{\infty} f(x) d x
$$

This will be amount to combine the size of Gate with probability $\mathrm{P}$. We also can find that the edge of Gate is related to $\chi_{\alpha}^{2}$ and the size of Gate depends on the size of error of $\sigma_{X}^{2}$ and $\sigma_{Y}^{2}$. We can get the critical point $\chi_{\alpha}^{2}$ according to the $\chi^{2}$ distribution table. If random variable $D^{2}$ is less than critical value $\chi_{\alpha}^{2}$, that means the second point and the first point are refection point of the same target. And the relation succeeds.

\section{Experimental Results of Data Fusion}

The data of Laser sensor LD-LRS3100 and LMS221 were collected, and be processed in space matching and data correlation and get the velocity and the length of ship according to the principal of speed testing.

We compare the characteristic value with the manual measurement value of 1500 ships combining with the extracted results of the width and the height above water in the third section. And we compute the error of the width, the length, the height of the ship respectively and we get a statistical chart of error as Figure 9. Shows.

And we can get the mean error of length $\varphi_{l}=5.32 \%$ and the mean error of height $\varphi_{h}=2.20 \%$ and the mean error of width $\varphi_{w} \varphi_{W=1.58 \% \text {. }}$

The error of length is bigger than the other two characteristic value. This is mainly because of the difference of the laser in performance. Laser sensor LD- LRS3100 have a better performance than Laser sensor LMS221 no matter in scanning frequency or angle resolution. And there is a little deviation when the two lasers are scanning the ship because of the instability of the ship track.

The system of vessel flow detection put into working in September 2012. Util now, it had a good performance both in stability and measurement accuracy.

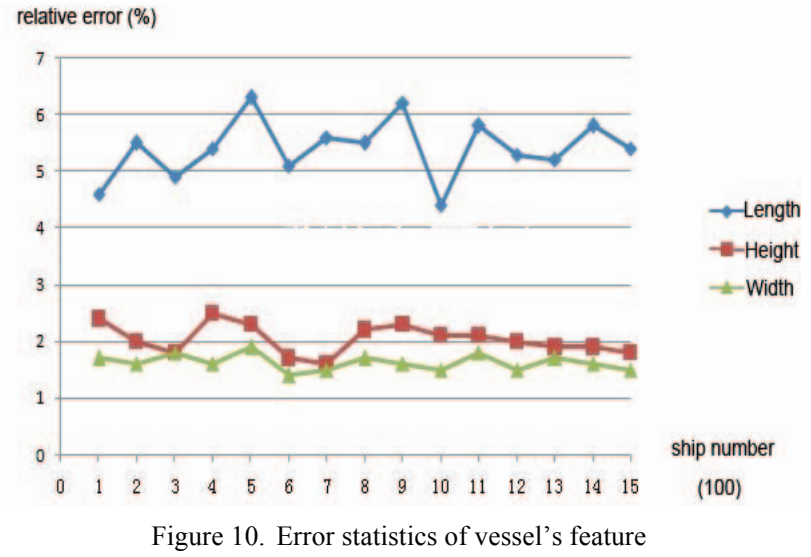

\section{CONCLUSION}

A method was proposed for ship contour feature extraction, based on mathematical morphology, expanding the contour, identifying the connected area based on Eight Code Chain. Contour reconfiguration makes a difference to the width of the ship in detail and proves the necessity and validity of reconfiguration.

Then an algorithm was studied to obtain the length detection using data-fusion and get the length of the ship by fusion computation of the space matched and data-related ship. We chose the data collected for one week and tested 1500 ships in practice and checked the error rate. The system of vessel flow detection worked fine.

\section{REFERENCES}

[1] Q. S. Jiang, "Detection de bateaux dans les images de radar a ouverture synthetique", Ph.D. dissertation, Univ. de Sherbrooke, Sherbrooke, CA, 2003.

[2] F. X. Zhu, "Estimation of ship capacity based on Computer Vision", Ph.D. dissertation, NanJing Univ. Sci. \& Tech., Nanjing, $\mathrm{CHN}$

[3] R. A. Steinberg and J. J. Rivera, "Infrared Ship Detection at Low Signal-to-Noise", Applied Physics Lab, Johns Hopkins Univ, MD, Rep: APL/JHU/FS-87-193, 68p, Sep 1987.

[4] H. Z. Chang, "The Study on the Accuracy of Shipborne AIS Information and the Establishment of Information Correction and Error Prewarning System", Ph.D. dissertation, Dalian Marit. Univ., Dalian, CHN, 2011.

[5] H. Cai, "Theroy and Application of Mathematical Morphology connectivity", Ph.D. dissertation, Zhejiang Univ, Hangzhou, CHN, 2009.

[6] S. S. Kim and J. H. Park, "Space-efficient terrain redering using constrained Delaunay trianay", in Geosci. Rem. Sens. Sym., 2002.

\section{AUTHORS}

W.Q. Li (corresponding author) is with School of Computer Science and Engineering, Nanjing University of Science and Technology, China, Nanjing, 210094; E-mail: li_weiqing@139.com

Y.T. Yang and S.H. Shen are with School of Computer Science and Engineering, Nanjing University of Science and Technology, Nanjing, China, 210094

Submitted 29 November 2013. Published as re-submitted by the authors 09 March 2014. 